Relations industrielles

Industrial Relations

\title{
Management de la flexibilité par Christophe EVERAERE, Paris : Économica, 1997, 203 p., ISBN 2-7178-3184-3.
}

\section{Bernard Galambaud}

Volume 53, numéro 2, printemps 1998

URI : https://id.erudit.org/iderudit/005278ar

DOI : https://doi.org/10.7202/005278ar

Aller au sommaire du numéro

Éditeur(s)

Département des relations industrielles de l'Université Laval

ISSN

0034-379X (imprimé)

1703-8138 (numérique)

Découvrir la revue

Citer ce compte rendu

Galambaud, B. (1998). Compte rendu de [Management de la flexibilité par

Christophe EVERAERE, Paris : Économica, 1997, 203 p., ISBN 2-7178-3184-3.]

Relations industrielles / Industrial Relations, 53(2), 371-373.

https://doi.org/10.7202/005278ar

Tous droits réservés @ C Département des relations industrielles de l'Université Laval, 1998
Ce document est protégé par la loi sur le droit d'auteur. L'utilisation des services d'Érudit (y compris la reproduction) est assujettie à sa politique d'utilisation que vous pouvez consulter en ligne.

https://apropos.erudit.org/fr/usagers/politique-dutilisation/ 


\section{Recension}

\section{Book Review}

\section{Management de la flexibilité}

par Christophe EvERAERE, Paris : Économica, 1997, 203 p., ISBN 2-7178-3184-3.

Les entreprises vivent sous la double contrainte de l'incertitude et de l'urgence pour reprendre les termes même utilisés par l'auteur. Et l'adaptation des entreprises à cette double contrainte est dans leur capacité à être flexible..

Devenir flexible, être flexible, telle est l'exigence du temps. La flexibilité est une exigence, et à ce titre, elle n'a pas à être discutée, elle s'impose, elle va de soit. "La capacité à réagir dans l'incertitude et dans l'urgence est une condition de survie... les entreprises rigides ont du souci à se faire " écrit l'auteur. Son propos est alors tout naturellement de l'ordre des moyens, de l'ordre du comment, abordé par les domaines classiques de la stratégie, de la production, des ressources humaines, des systèmes d'information et du contrôle de gestion.

Dans un ouvrage déjà ancien, Julienne Brabet (Repenser la gestion des ressources humaines ? Paris : Économica, 1993) soulignait combien toute une part de la littérature de gestion reposait sur des postulats optimistes, sur la conviction que les acteurs sociaux avaient en fait des intérêts communs. Christophe Everaere s'inscrit dans cette ligne. Son intention est nette : "À certaines conditions, le management de la flexibilité, pour les individus... peut recouvrir des aspects tout à fait positifs... Toute une série de considérations qui peuvent faire de la flexibilité un jeu à somme positive, à la fois pour les entreprises et pour les individus ". Gagnant, gagnant, c'est encore possible. Il faut d'ailleurs que cela le soit ! Telle semble être la conviction d'un auteur qui ne désespère pas de réconcilier une fois encore l'économie et l'humanisme. Mais le management n'est-ce pas d'abord et avant tout, des convictions, des croyances instrumentées?

À tout seigneur, tout honneur : la stratégie! Ce qui est stratégique dans l'entreprise flexible, c'est justement de se donner les moyens de réactions à l'événement. Et dans ces moyens là, les hommes et leurs capacités, leurs compétences, leurs talents, leur imagination tiennent une place majeure; les hommes et leur management engagés dans des processus participatifs et consensuels. Mais cette participation là n'est pas sereine.

Ce consensus là, n'est pas un consensus mou, fondé sur la complaisance partagée et la crainte du conflit. Au contraire, participations et consensus sont faits de confrontations qui peuvent être rugueuses, mais au combien nécessaires à l'identification des problèmes et à l'émergence de leurs solutions dans un apprentissage collectif. Cette position de l'auteur rencontre celle d'auteurs comme Philippe Zarifian ou Pierre Leclair (L'acteur et le débat, février 1996, Entreprise \& Personnel) qui voient dans le débat, au sein des collectifs de travail, une source essentielle du développement des com- 
pétences dans la mesure ou celles-ci ne sont pas seulement savoir faire mais aussi engagement. La compétence est aussi une attitude, une position... que le salarié peut adopter ou refuser d'adopter.

L'auteur dénonce le mythe de l'usine sans hommes, le mythe de la modernité de tous les dirigeants qui ont fait sur l'homme au travail un pari pessimiste ; pessimisme qui a engendré leur fantasme du " tout automatique "... Ils ont voulu des machines intelligentes et le monde qu'ils ont créé est devenu inintelligible, tant pour autrui que pour eux-mêmes

L'auteur s'en prend également à la théorie des coûts de transactions qui est marquée à ses yeux des mêmes présupposés négatifs, présupposés que Taylor avait déjà fait siens. Aussi, Christophe Everaere préfère-t-il la notion d'investissement de transaction. Perdre du temps à prendre collectivement une décision en intégrant dans le processus le plus d'acteurs possibles, c'est, en dernière analyse, en gagner. Cette perte de temps qui indispose le "décideurminute " est en fait plus efficace que la majeure partie des décisions prises rapidement de façon centralisée et autoritaire. Les exemples ne manquent pas de ces décisions prises en quelques instants dans le silence du cabinet et qui s'éternisent dans leur mise en oeuvre... Ne vaut-il pas mieux un processus de décision plus lent, mais aussi plus capable d'engendrer une action rapide. Mais, pour cela, il faut cesser d'être fasciné par le mythe du " décideur »...

Comment espérer gérer l'incertitude et l'urgence sans obtenir la participation active et l'adhésion du personnel au processus d'adaptabilité ? L'auteur dénonce en fait " un management par la suspicion et la méfiance " et appelle de ses voeux un "management par la confiance ". Christophe Everaere s'amuse à souligner une curiosité de langage très révélatrice: l'on dit les machines et ateliers flexibles et les hommes sont qualifiés de polyvalents. Or, en vérité, ce sont les machines et ateliers qui sont au mieux polyvalents alors que les hommes ont effectivement la capacité d'être flexibles grâce à l'apprentissage, à la mémorisation, à la réflexion, à l'intuition... Il n'en reste pas moins que pour l'auteur cette anecdote langagière est révélatrice d'une pensée dominante qui espère plus en la machine qu'en l'homme.

Bien sur, l'optimisme de Christophe Everaere n'est pas sans limite, n'est pas universel. Il sait bien, notamment, que les pratiques de gestion des ressources humaines sont contingentes, en partie, aux organisations qu'elles servent.

Mais, lorsqu'il convient de faire face à la diversité, à l'incertitude, à l'urgence, alors l'entreprise a besoin de personnel compétent, responsable, autonome, capable de faire face aux dysfonctionnements et aléas et capable de saisir les opportunités. Aussi, plutôt que d'opter pour une flexibilité quantitative ou les salariés ne sont qu'une variable d'ajustement et qui engendre chez ces derniers précarité et déqualification, plus d'une entreprise serait plus inspirée d'opter pour une flexibilité qualitative. C'est pourquoi l'auteur croit que l'heure de ce qu'il nomme le modèle de la compétence renouant le lien entre réflexion et exécution, que Taylor et ses successeurs avaient tranché, est d'actualité. Au lieu de tourner leur pessimisme vers l'homme, plus d'un dirigeant ferait bien de le tourner vers la machine. Aujourd'hui, la " flânerie des machines " peut être plus coûteuse que celle des hommes... comme les pannes des robots plus coûteuses que les grèves des hommes.

Mais ce salarié compétent, responsable, engagé, il faut le rémunérer en conséquence. Et cela ne doit pas poser un vrai problème. Un salarié compétent et autonome n'a pas besoin du même 
taux d'encadrement, est capable d'une meilleure productivité... Bref, comme le dit l'auteur, il est absurde de rogner sur les charges salariales lorsque "les opérateurs ont dans les mains des millions de francs d'équipements... ".

Enfin, l'auteur affirme vigoureusement qu'il n'y a pas de flexibilité réelle, efficace, de l'entreprise sans stabilité de l'emploi et sans intégration du personnel. Les compétences se sont toujours construites dans la durée, dans l'entreprise ou dans son métier. La mobilité n'est pas un vagabondage ; la mobilité n'est pas une errance. Les hommes peuvent être une ressource stratégique déterminante dans le management de la flexibilité, d'autres dispositions doivent être prises par le management en matière, notamment, de système d'information et de contrôle de gestion. Ce dernier doit rompre avec une vision mécanique pour une approche dyna- mique d'implication des acteurs dans une relation de confiance. Ce qui importe alors plus que le constat du résultat est la maîtrise de l'orientation et des processus de décisions et d'actions.

Bref, ce livre souligne avec force que face aux nouvelles exigences du fonctionnement d'une entreprise, il y a place pour les choix des dirigeants. Ils peuvent choisir de se méfier des hommes et de leurs coûts ; ils peuvent choisir au contraire leur capacité à créer, à imaginer, à inventer, à s'adapter... Ce livre souligne également avec force que si la compétence du salarié est bien un engagement, une attitude, il n'en est pas différemment de la compétence manageriale.

BERNARD GALAMBAUD

ESCP-Paris et Entreprise \& Personnel 